\title{
Hakuna Nematoda: genetic and phenotypic diversity in African isolates of Caenorhabditis elegans and $C$. briggsae
}

\author{
ES Dolgin ${ }^{1}$, M-A Félix ${ }^{2}$ and AD Cutter ${ }^{1,3}$ \\ ${ }^{1}$ Institute of Evolutionary Biology, University of Edinburgh, King's Buildings, Edinburgh, UK; ${ }^{2}$ Institut Jacques Monod, Centre National \\ de la Recherche Scientifique, Universities of Paris, Paris, France and ${ }^{3}$ Department of Ecology and Evolutionary Biology, University of \\ Toronto, Toronto, Ontario, Canada
}

\begin{abstract}
Caenorhabditis elegans and C. briggsae have many parallels in terms of morphology, life history and breeding system. Both species also share similar low levels of molecular diversity, although the global sampling of natural populations has been limited and geographically biased. In this study, we describe the first cultured isolates of $C$. elegans and C. briggsae from sub-Saharan Africa. We characterize these samples for patterns of nucleotide polymorphism and vulva precursor cell lineage, and conduct a series of hybrid crosses in $C$. briggsae to test for genetic incompatibilities. The distribution of genetic diversity confirms a lack of geographic structure to $C$. elegans sequences but shows genetic differentiation of $C$. briggsae into three distinct clades that may correspond to three latitudinal ranges. Despite low
\end{abstract}

levels of molecular diversity, we find considerable variation in cell division frequency in African $C$. elegans for the P3.p vulva precursor cell, and in African C. briggsae for P4.p, a variation that was not previously observed in this species. Hybrid crosses did not reveal major incompatibilities between C. briggsae strains from Africa and elsewhere, and there was some evidence of inbreeding depression. These new African isolates suggest that important ecological factors may be shaping the patterns of diversity in $C$. briggsae, and that despite many similarities between $C$. elegans and C. briggsae, there may be more subtle differences in their natural histories than previously appreciated.

Heredity (2008) 100, 304-315; doi:10.1038/sj.hdy.6801079; published online 12 December 2007

Keywords: Caenorhabditis elegans; Caenorhabditis briggsae; genetic variation; vulva; self-fertilization; androdioecy

\section{Introduction}

As a model organism, the nematode Caenorhabditis elegans has provided an outstanding system for elucidating fundamental problems in biology. However, the attitude regarding the natural history and ecology of Caenorhabditis has long been 'hakuna matata', to use the Swahili phrase meaning 'no worries'. To achieve a robust understanding of the evolutionary context in which $C$. elegans' developmental and genetic patterns emerged, it is necessary to characterize representative natural populations on a worldwide scale and to conduct comparative analyses of Caenorhabditis species. Specifically, the quantification of genetic and phenotypic variation in species with shared common ancestry permits inference about the evolutionary forces and population processes that shape the life history and development of these organisms.

Along with $C$. elegans, C. briggsae forms part of a monophyletic clade of five species in laboratory culture known as the Elegans group of the genus Caenorhabditis (Kiontke and Fitch, 2005). Like C. elegans, C. briggsae is

Correspondence: ES Dolgin, Institute of Evolutionary Biology, School of Biological Sciences, University of Edinburgh, King's Buildings, Edinburgh, EH9 3JT, UK.

E-mail: elie.dolgin@ed.ac.uk

Received 29 June 2007; revised 8 October 2007; accepted 8 November 2007; published online 12 December 2007 androdioecious (self-fertile hermaphrodites and facultative males), but both species evolved independently from gonochoristic (male-female) ancestors (Kiontke et al., 2004). Despite analogous breeding systems and similar morphology (Nigon and Dougherty, 1949), their genome sequences have diverged drastically (Coghlan and Wolfe, 2002; Stein et al., 2003), although they do show a high degree of chromosomal synteny (Hillier et al., 2007). The development of $C$. briggsae as a laboratory system is still in its infancy, but with a growing number of genetic resources becoming available, including a sequenced genome and recombination maps, this species is emerging as a useful companion species of C. elegans (Baird and Chamberlin, 2006).

C. elegans and C. briggsae are cosmopolitan species with overlapping geographic distributions, with at least one of the two species found on each continent where intensive field collections have been carried out. Both species show similarly low levels of genetic variation, especially when compared to the related gonochoristic species, C. remanei (Graustein et al., 2002; Jovelin et al., 2003; Haag and Ackerman, 2005; Cutter, 2006; Cutter et al., 2006a, b). The predominantly self-fertilizing breeding systems shared by both $C$. elegans and $C$. briggsae has probably driven this decrease in diversity as a consequence of reduced effective population size, increased homozygosity and greater population subdivision (Charlesworth, 2003). Despite their similarities, however, C. elegans and 
C. briggsae probably occupy different ecological niches. For example, one difference relevant to ecology is that $C$. briggsae tolerates and proliferates at higher temperatures than C. elegans (Fodor et al., 1983). C. elegans is also strongly induced to form the dormant dauer larval stage at high temperatures, whereas C. briggsae appears to lack this response (Inoue et al., 2007).

Another difference of potential ecological significance is the relative importance of geography in structuring genetic variation. Phylogenetic reconstructions of molecular polymorphisms among available global samples show no strong signature of geographic structure in $C$. elegans (Denver et al., 2003; Barrière and Félix, 2005; Haber et al., 2005; Cutter, 2006), but a clear division is observed in $C$. briggsae between strains from temperate latitudes and strains found near the Tropic of Cancer (Graustein et al., 2002; Cutter et al., 2006b). A major problem with our current understanding of natural variation and biogeography, however, is that it is unclear whether the focal samples adequately reflect global diversity, because sampling efforts have not targeted most regions outside North America and Europe. Although the genus was first described from specimens isolated in North Africa (Maupas, 1900), these strains were never cultured, and, to date, no C. elegans or C. briggsae strains have been analyzed from Africa or South America. To address this issue, one of us (ESD) undertook intensive field collections in two countries in sub-Saharan Africa: Kenya and South Africa.

In this study, we test the hypotheses concerning geographic structuring of genotypes in C. elegans and C. briggsae with new samples from Africa. We also examine vulval cell lineages for phenotypic polymorphisms and conduct intraspecific crosses in C. briggsae to test for genetic incompatibilities. Our results confirm the lack of global geographic structure in the sequences of $C$. elegans, but provide further evidence for latitudinal clades in C. briggsae. We also find unique patterns of vulval cell division in South African strains of C. briggsae that have not been observed previously in this species, while crosses between isolates from disparate localities fail to demonstrate hybrid incompatibilities (Dolgin et al., 2007), and instead show evidence of inbreeding depression. We discuss how these new isolates add to our understanding of global variation and how evolutionary processes might act differently on the two species after their independent transitions to androdioecy.

\section{Methods}

\section{Nematode populations}

We isolated 38 new strains of C. elegans and 25 new strains of $C$. briggsae from single wild hermaphrodites in Kenya and South Africa in March-April 2006. Despite extensive sampling from 36 sites, Caenorhabditis nematodes were only found in four locations: (1) compost from a private garden (Johannesburg, South Africa); (2) compost from a plant nursery (Ceres, South Africa); (3) compost from a mushroom farm (Limuru, Kenya) and (4) leaf litter in a public park (Nairobi, Kenya). Strain name designations for C. elegans are ED3040-ED3077, and for C. briggsae are ED3078-ED3102. The sampling sites and strain designations are described in Table 1 . Various other nematode species were also found in these sites, including many strains of Oscheius tipulae, which have also been characterized (Baille et al., 2008).

The sampling protocol was carried out using the 'Isolation on an Agar Plate' technique as described by Barrière and Félix (2006). Briefly, small samples of compost or leaf litter were placed on 9-cm NGM-lite agar plates spotted with Escherichia coli OP50. These plates were then monitored for nematodes crawling out into the bacterial lawn for $24-48 \mathrm{~h}$, and individual worms were isolated onto separate $6-\mathrm{cm}$ plates to establish independent iso-hermaphrodite lineages. Self-fertile individuals were examined for morphological features under compound microscopy, and species identity was made through mating tests and by sequencing a portion of the small subunit ribosomal RNA gene (Floyd et al., 2002).

For DNA sequencing, we randomly chose a subset of 16 strains of each species from the African collection as representative of these sampling sites (Table 1). The sequences from these strains were then compared to isolates from a larger worldwide data set of 118 C. elegans strains (Cutter, 2006) and 63 C. briggsae strains (Cutter et al., 2006b). To gain a more complete understanding of the molecular diversity in the two species, we also used an additional nine strains that were recently isolated from geographically unique locations and had not previously been assessed for nucleotide polymorphism: a C. elegans strain from Madagascar (LKC34; isolated by V. Stowell and donated by L. Carta), a C. elegans strain from Ribeiro Frio, Madeira (JU258; isolated by MAF), a C. elegans strain from Kakegawa, Japan (JU1088; isolated by MAF), two $C$. elegans strains from Concepcion, Chile (JU1171 and JU1172; isolated by MAF), a C. briggsae strain from Kruger National Park, South Africa (DF5100; isolated by $\mathrm{W}$ Sudhaus and donated by K Kiontke), a C. briggsae strain from Kakegawa, Japan (JU1085; isolated by MAF) and two C. briggsae strains from Salt Lake City, UT, USA (EG4181 and EG4207; isolated by M Ailion and obtained from the Caenorhabditis Genetics Center). The geographic origin of each C. elegans and C. briggsae strain included in this analysis is shown in Figure 1.

\section{Molecular methods}

DNA from single individuals was obtained using a $\mathrm{NaOH}$ digestion protocol (Floyd et al., 2002). We sequenced the gene fragments that had previously been analyzed for a larger global collection of strains (Cutter, 2006; Cutter et al., 2006b). These comprised six genes from C. elegans chromosomes II and X, and the putative orthologs of five of these genes plus one locus nearby the sixth gene in C. briggsae, with primers designed to span a long (>500 bp) intron. Names, lengths, positions and primers of the sequenced loci can be found as described by Cutter (2006) and Cutter et al. (2006b). Both strands were sequenced on an ABI Prism 3730 automated sequencer at the School of Biological Sciences Sequencing Service, University of Edinburgh.

\section{Sequence analysis}

Sequence alignment and manual editing to remove the primers were performed with Sequencher 4.6 and BioEdit 7.0.5. Calculations of diversity (from pairwise differences, $\pi$, and from the number of segregating sites, $\theta)$, Tajima's $D$ tests of neutrality (Tajima, 1989), and tests 
Table 1 New C. elegans and C. briggsae strains isolated in Kenya and South Africa, and a list of the strains used for DNA sequencing

\begin{tabular}{|c|c|c|c|c|c|c|c|c|c|}
\hline \multirow[t]{2}{*}{ Location (nearest city) } & \multirow{2}{*}{$\begin{array}{l}\text { Latitude } \\
\text { (S) }\end{array}$} & \multirow{2}{*}{$\begin{array}{l}\text { Longitude } \\
\text { (E) }\end{array}$} & \multirow{2}{*}{$\begin{array}{l}\text { Altitude } \\
(m)\end{array}$} & \multicolumn{3}{|c|}{ C. elegans } & \multicolumn{3}{|c|}{ C. briggsae } \\
\hline & & & & No. & $\begin{array}{c}\text { Strain } \\
\text { designations }\end{array}$ & $\begin{array}{l}\text { Strains used } \\
\text { in sequencing } \\
\text { (and haplotypes) }\end{array}$ & No. & $\begin{array}{c}\text { Strain } \\
\text { designations }\end{array}$ & $\begin{array}{l}\text { Strains used } \\
\text { in sequencing }\end{array}$ \\
\hline Johannesburg, South Africa & $26^{\circ} 10^{\prime}$ & $28^{\circ} 01^{\prime}$ & 1669 & 1 & ED3040 & ED3040 $(\alpha)$ & 12 & ED3078-ED3089 & $\begin{array}{l}\text { ED3078 } \\
\text { ED3079 } \\
\text { ED3080 } \\
\text { ED3082 } \\
\text { ED3083 } \\
\text { ED3084 } \\
\text { ED3087 } \\
\text { ED3089 }\end{array}$ \\
\hline Ceres, South Africa & $33^{\circ} 22^{\prime}$ & $19^{\circ} 19^{\prime}$ & 478 & 12 & ED3041-ED3052 & $\begin{array}{l}\text { ED3042 }(\beta) \\
\text { ED3046 }(\gamma) \\
\text { ED3048 }(\beta) \\
\text { ED3049 }(\gamma) \\
\text { ED3051 }(\beta) \\
\text { ED3052 }(\delta)\end{array}$ & 0 & & \\
\hline Limuru, Kenya & $1^{\circ} 05^{\prime}$ & $36^{\circ} 39^{\prime}$ & 2356 & 24 & ED3053-ED3076 & $\begin{array}{l}\text { ED3054 }(\varepsilon) \\
\text { ED3057 }(\varepsilon) \\
\text { ED3063 }(\varepsilon) \\
\text { ED3066 }(\varepsilon) \\
\text { ED3072 }(\varepsilon) \\
\text { ED3073 }(\varepsilon) \\
\text { ED3075 }(\varepsilon) \\
\text { ED3076 }(\varepsilon)\end{array}$ & 0 & & \\
\hline Nairobi, Kenya & $1^{\circ} 19^{\prime}$ & $36^{\circ} 48^{\prime}$ & 1708 & 1 & ED3077 & ED3077 ( $\beta)$ & 13 & ED3090-ED3102 & $\begin{array}{l}\text { ED3091 } \\
\text { ED3092 } \\
\text { ED3095 } \\
\text { ED3096 } \\
\text { ED3098 } \\
\text { ED3099 } \\
\text { ED3100 } \\
\text { ED3101 }\end{array}$ \\
\hline
\end{tabular}

C. elegans haplotype designations are as shown in Figure 2a.

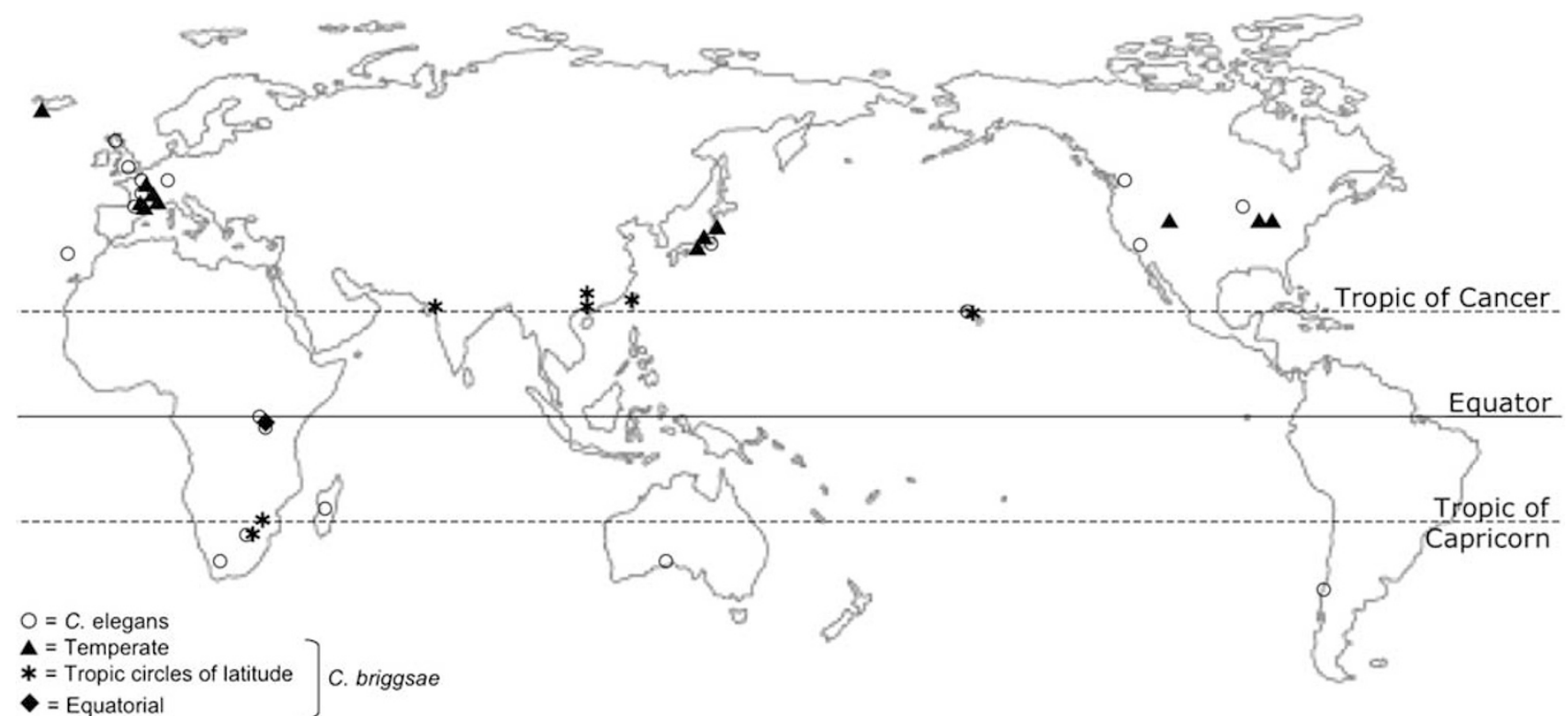

Figure 1 Geographic distribution of strains used for molecular analysis. Open circles indicate C. elegans strains and closed symbols indicate C. briggsae strains from different latitudinal clades: triangles (temperate latitude samples), asterisks (Tropic circles of latitude samples) and diamonds (equatorial samples). Note that the exact sampling location of C. elegans strain LKC34 is uncertain, although it is known to originate from Madagascar. 
of population differentiation using average values of $K_{\mathrm{s}}^{*}$ among loci (Hudson et al., 1992) and Hudson's (Hudson, 2000) nearest-neighbor statistic, $S_{\mathrm{nn}}$, from concatenated sequences were made using DnaSP 4.10.9 (Rozas et al., 2003). We present diversity data from silent sites (synonymous and intronic positions); sites corresponding to indels or incomplete data were excluded from the analyses. Neighbor networks and neighbor-joining trees were constructed with concatenated sequences using SplitsTree 4.6 (Huson and Bryant, 2006).

\section{Vulval cell lineage}

The frequency of division of the vulval precursor cells, P3.p and P4.p, was measured in a subset of the new African isolates-five C. elegans strains (ED3040, ED3046, ED3052, ED3054 and ED3077) and four C. briggsae strains (ED3082, ED3087, ED3092 and ED3101) - in addition to C. elegans strains JU258 and LKC34. The results were compared to a global collection of $13 \mathrm{C}$. elegans strains and $6 \mathrm{C}$. briggsae strains that had previously been scored for these polymorphic phenotypes (Delattre and Félix, 2001). For some of these previously analyzed strains, more worms were also scored to obtain larger sample sizes. We also screened eight strains from other species in the Elegans group of the Caenorhabditis genus for comparison-four strains of C. remanei (JU724, JU825, PB4641 and SB146), two strains of C. brenneri (CB5161 and PB2801) and two strains of Caenorhabditis sp. 5 (JU727 and SB378). The fate of the Pn.p cells was assessed at the L4 stage using Nomarski miscroscopy under standard conditions. We tested for heterogeneity between intraspecific strains by using replicated goodness-of-fit tests on the number of individuals in which the Pn.p cell divided or not, and performed post hoc pairwise tests after applying the Dunn-Šidák correction (Sokal and Rohlf, 1995, p. 239).

\section{Hybrid crosses}

To test for possible incompatibilities between strains of $C$. briggsae, a series of crosses were set up using strains from South Africa (ED3083) and Kenya (ED3101), as well as three additional C. briggsae strains: AF16 from India, HK104 from Japan and ED3034 from Taiwan. Pairwise crosses to the two African strains created seven crosses: ED3083 × ED3034, ED3083 × AF16, ED3083 × HK104, ED3083 $\times$ ED3101, $\quad$ ED3101 $\times$ ED3034, ED3101 $\times$ AF16 and ED3101 $\times$ HK104. We also performed an eighth cross between AF16 and HK104 since this interstrain cross has been studied previously (Baird et al., 2005). Hybrid crosses were only performed in C. briggsae in this study because only African samples of this species showed markedly different molecular and developmental patterns (see the Results), and hybrid crosses with representative populations have been performed elsewhere for C. elegans (Dolgin et al., 2007).

In each cross, we generated mixed-mating populations of males $(\mathrm{M})$ and hermaphrodites $(\mathrm{H})$ for each strain. We then established pure-strain F1s from each strain (for example, ED3101 $\times$ ED3101 and AF16 $\times$ AF16), and the two reciprocal F1 hybrids (for example, ED3101$\mathrm{M} \times \mathrm{AF} 16-\mathrm{H}$ and AF16-M $\times$ ED3101-H). We then measured the number of surviving late larval F2s (to give a measure of F1 brood size), the percentage of F2 embryonic lethality and the developmental timing to reach the L4 larval stage in the F2s. Interstrain crosses of $C$. briggsae were performed using the methods that Dolgin et al. (2007) used for C. elegans, with a few notable exceptions. Following synchronization of the pure-strain parental worms using alkaline hypochlorite, some L4 hermaphrodites were transferred to a new plate and the remaining worms were left with the males in a mixed-sex population. The isolated hermaphrodites were permitted to self for $\sim 3.5$ days following first egg-lay to exhaust them of self-sperm. Next generation males were then taken from the mixed-sex population and were mated to these sperm-depleted hermaphrodites for a $24 \mathrm{~h}$ interval. The mated hermaphrodites were subsequently transferred to new plates to lay eggs for $5 \mathrm{~h}$. From these F1 progeny, many L4 hermaphrodites of each pure strain or hybrid genotype (mean number $=19.2 \pm 1.1$ s.e.) were set up individually to measure surviving larval brood size and embryonic lethality. These worms were transferred every $24 \mathrm{~h}$ to a new plate; $36 \mathrm{~h}$ after transfer, unhatched eggs and surviving larvae were counted. Subsets of the remaining F1 hermaphrodites were transferred en masse to new plates at the L4 stage. Several hours after eggs were observed on these plates, the worms were transferred twice again to new plates to lay eggs for two $1 \mathrm{~h}$ intervals. These plates were then monitored for when the F2 progeny molted from the L4 stage to adulthood, as a measure of developmental timing. The mean brood sizes and levels of embryonic lethality within the same hybrid cross were compared using $t$-tests. Differences in developmental timing were evaluated using goodness-of-fit tests on the number of 'normal' or 'late' molting larvae, where late was defined as more than $5 \mathrm{~h}$ after the peak molting time.

\section{Results}

\section{Molecular polymorphism}

The African sample of C. elegans shows similar low levels of within-locality variation as reported previously for other regions of the world. Overall silent-site nucleotide polymorphism in the African localities was equivalent to the European samples, with Africa-wide $\pi_{\mathrm{si}}$ and $\theta_{\mathrm{si}}$ both estimated to be $\sim 0.2 \%$ (Table 2 ). Considering diversity levels of South African or Kenyan isolates independently also resulted in quantitatively similar diversity levels to that found in individual European localities. Despite low levels of variation, there is no evidence of non-neutral demographic or selective processes in the African samples, either as a whole or by considering each country individually, as there was a range of positive and negative values of Tajima's $D$ across the different loci, with no values significantly different from zero (results not shown).

The 17 African samples of C. elegans (including LKC34 from Madagascar) contained six different haplotypes. Although these haplotypes were endemic to African samples, most polymorphic sites are also found elsewhere in the world (Figure 2a). All the African strains were generally similar to other strains and showed no strong patterns of geographic structure (Figure 3a and Supplementary Figure S1a); however, we did find some evidence of population differentiation between European and African samples $\left(K_{\mathrm{st}}^{*}=0.075 ; S_{\mathrm{nn}}=0.97, P<0.0001\right)$ and between Kenyan and South African samples 
Table 2 Summary of nucleotide polymorphisms for African C. elegans and C. briggsae isolates

\begin{tabular}{|c|c|c|c|c|c|c|c|c|c|c|c|}
\hline \multirow[t]{2}{*}{ Locus (chromosome) } & \multicolumn{7}{|c|}{ African population samples } & \multicolumn{2}{|c|}{ South Africa } & \multicolumn{2}{|c|}{ Kenya } \\
\hline & Silent sites & $S$ & $h$ & $H_{d}$ & Indels & $\pi_{s i}$ & $\theta_{s i}$ & $\pi_{s i}$ & $\theta_{s i}$ & $\pi_{s i}$ & $\theta_{s i}$ \\
\hline \multicolumn{12}{|l|}{ C. elegans } \\
\hline Y25C1A.5 (II) & 658.8 & 7 & 4 & 0.596 & 4 & 0.0036 & 0.0031 & 0.0033 & 0.0031 & 0.0013 & 0.0022 \\
\hline ZK430.1 (II) & 542.8 & 1 & 2 & 0.529 & 1 & 0.0010 & 0.0005 & 0 & 0 & 0.0004 & 0.0007 \\
\hline E01G4.6 (II) & 521.4 & 16 & 3 & 0.324 & 2 & 0.0066 & 0.0091 & 0.0092 & 0.0125 & 0 & 0 \\
\hline D1005.1 (X) & 480.0 & 0 & 1 & 0 & 0 & 0 & 0 & 0 & 0 & 0 & 0 \\
\hline R160.7 (X) & 605.2 & 0 & 1 & 0 & 0 & 0 & 0 & 0 & 0 & 0 & 0 \\
\hline T24D11.1 (X) & 651.0 & 1 & 2 & 0.309 & 0 & 0.0005 & 0.0005 & 0.0007 & 0.0006 & 0 & 0 \\
\hline Concatenated & 3459.2 & 25 & 6 & 0.743 & 7 & 0.0019 & 0.0021 & 0.0021 & 0.0026 & 0.0003 & 0.0005 \\
\hline Average & 576.5 & 4.2 & 2.2 & 0.293 & 1.2 & 0.0019 & 0.0022 & 0.0022 & 0.0027 & 0.0003 & 0.0005 \\
\hline \multicolumn{12}{|l|}{ C. briggsae } \\
\hline p09 & 484.7 & & & & & & & 0 & 0 & 0 & 0 \\
\hline p10 & 581.3 & & & & & & & 0.0004 & 0.0006 & 0.0004 & 0.0007 \\
\hline p11 & 571.3 & & & & & & & 0 & 0 & 0 & 0 \\
\hline p12 & 623.7 & & & & & & & 0.0007 & 0.0012 & 0 & 0 \\
\hline p13 & 593.5 & & & & & & & 0 & 0 & 0 & 0 \\
\hline p14 & 599.5 & & & & & & & 0 & 0 & 0 & 0 \\
\hline Concatenated & 3452.0 & & & & & & & 0.0002 & 0.0003 & 0.0001 & 0.0001 \\
\hline Average & 575.7 & & & & & & & 0.0002 & 0.0003 & 0.0001 & 0.0001 \\
\hline
\end{tabular}

Abbreviations: $\mathrm{S}$, segregating sites; $\mathrm{h}$, haplotypes; $\mathrm{H}_{\mathrm{d}}$, haplotype diversity; $\pi_{\mathrm{si}}$, silent-site diversity from pairwise differences; $\theta_{\mathrm{si}}$, silent-site diversity from the number of segregating sites.

Diversity estimates were calculated for each country in both species, and for all African samples for C. elegans but not for C. briggsae due to the distinct geographical structuring between countries in this species. For C. elegans, there were a total of 17 strains - 7 from South Africa, 9 from Kenya and 1 from Madagascar (LKC34). For C. briggsae, there were a total of 17 strains -9 from South Africa (including DF5100) and 8 from Kenya.

$\left(K_{\mathrm{st}}^{*}=0.215 ; \quad S_{\mathrm{nn}}=0.85, \quad P=0.003\right)$. Nonetheless, after including the African samples, we did not find evidence of any additional recombination events to those found previously, as indicated by the four-gamete test (Hudson and Kaplan, 1985). We also sequenced loci for the first known C. elegans isolates from Asia (JU1088 from Japan) and South America (JU1171 and JU1172 from Chile), plus the Madeiran strain JU258, since it was previously reported that this strain was highly differentiated from other strains (Haber et al., 2005; Stewart et al., 2005). Despite the unique geographical origin of these additional strains, we failed to find high divergence of any of them from global samples for these six loci (see haplotype Y for JU1088, haplotype I for JU1171, haplotype K for JU1172 and haplotype Z for JU258, in Figures $2 \mathrm{a}$ and $3 \mathrm{a}$ ). Furthermore, JU258 was not strongly allied to the Hawaiian strain, CB4856 (haplotype T), relative to the $\mathrm{N} 2$ strain (haplotype A), as previously suggested (Haber et al., 2005; Stewart et al., 2005; Maydan et al., 2007).

The African C. briggsae samples show a markedly different pattern from that found for C. elegans. We observed very little within-locality diversity across the six loci tested, with South African and Kenyan $\pi_{\mathrm{si}}$ and $\theta_{\mathrm{si}}$ estimated to be extremely low (Table 2). This results from the fact that seven of eight Kenyan strains were identical, with only a single nucleotide polymorphisms (SNP) distinguishing ED3092, and of the nine South African strains, the eight strains from Johannesburg were identical to each other, and DF5100, from Kruger National Park, had only three SNP differences from the Johannesburg strains. Nonetheless, the African C. briggsae haplotypes were very informative about geographic structure (Figure 2b). The South African haplotypes were remarkably similar to those of other strains found in the northern hemisphere near the Tropic of Cancer, and the Kenyan haplotypes were unlike those of any other strains previously described, with many newly identified polymorphisms not shared with other populations (Figure 3b and Supplementary Figure S1b). For example, the p11 locus had previously been shown to be monomorphic (Cutter et al., 2006b), but we found seven SNPs and three indels in the Kenyan samples (Figure 2b). However, since most of these polymorphisms were unique to this locality, we found no further evidence of historical recombination after including the African samples, as indicated by the four-gamete test (Hudson and Kaplan, 1985).

The 'tropical' strains previously described originated from China, India and Hawaii-all situated within $3^{\circ}$ of the Tropic of Cancer. The South African strains described here were isolated in Johannesburg, less than $3^{\circ}$ south of the Tropic of Capricorn, and Kruger National Park, which straddles the Tropic of Capricorn. Considering that these samples were all quite different from the Kenyan samples, which is also technically a tropical location, we propose that what was previously called 'tropical' strains (Cutter et al., 2006b) should be considered as 'Tropic circles of latitude' strains, and suggest that molecular diversity in C. briggsae strains may be partitioned into three major latitudinal clades: temperate samples, Tropic circles of latitude samples and equatorial 

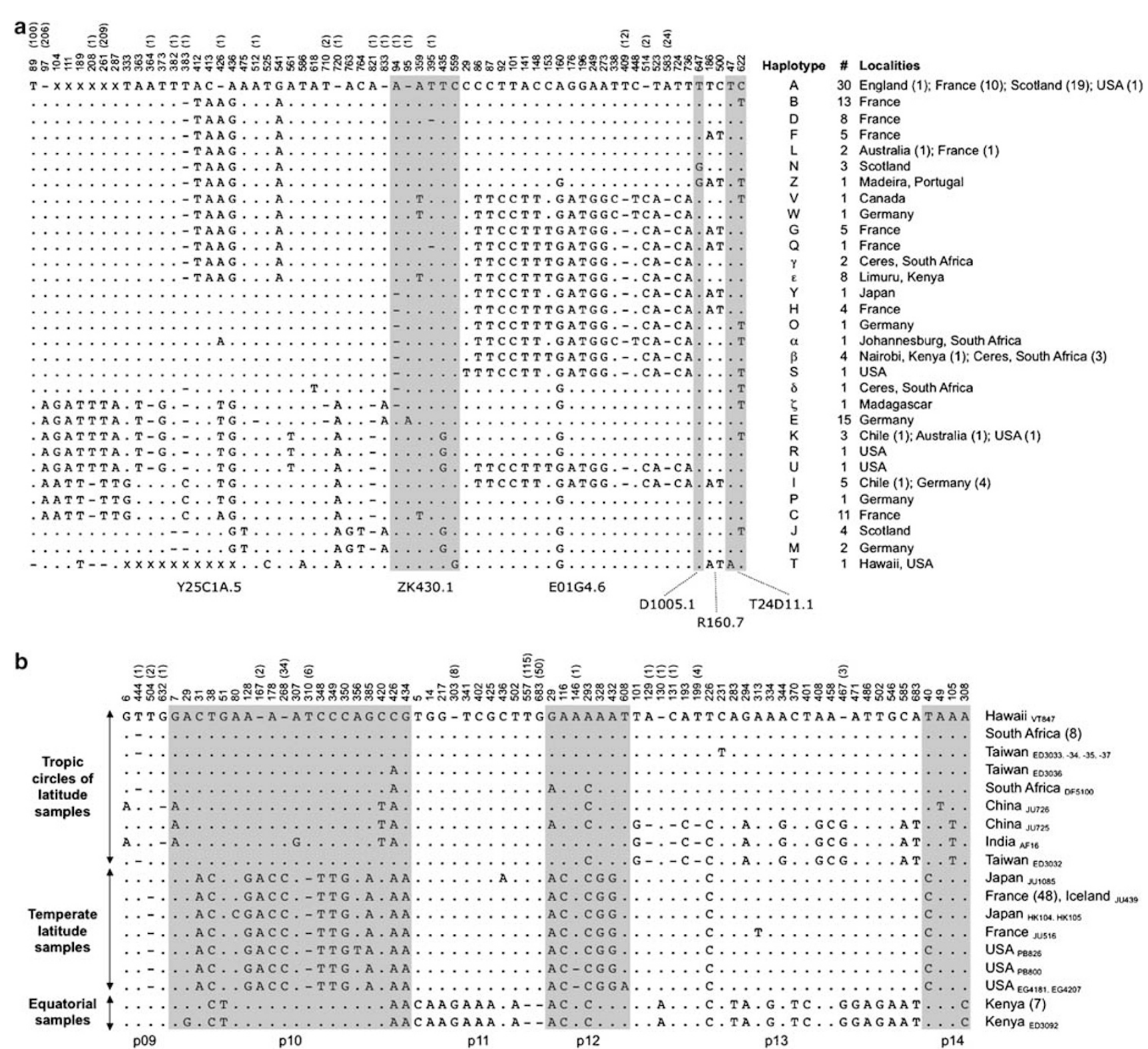

Figure 2 Summary of multilocus haplotypes for C. elegans (a) and C. briggsae (b). Loci are labeled along the bottom, with the positions of polymorphisms relative to the start of each locus indicated across the top (indel lengths in parentheses). For $C$. elegans, the haplotype designations follow those described by Cutter (2006), with JU1088 denoted by haplotype Y, JU258 denoted by haplotype Z and the African haplotypes indicated by Greek letters. For a list of which strains correspond to each African haplotype, see Table 1 . Haplotype X described by Cutter (2006) was found to be equivalent to A, as a consequence of an alignment error at position 426 in Y25C1A.5. We also amend several presentation errors from Figure 1 as described by Cutter (2006).

samples (Figures $2 b$ and $3 b$ ). An alternative model of geographic structure is that a variety of deeply divergent clades of $C$. briggsae populate the globe in a manner that only mimics a latitudinal distribution, as a consequence of available sampling. We also sequenced three additional recently isolated strains: a strain from Kakegawa, Shizuoka Prefecture, Japan (JU1085) and two from Salt Lake City, UT, USA (EG4181 and EG4207), and observed that these strains were quite similar to other strains from temperate latitudes, consistent with the prediction of a temperate latitudinal clade (see Figures $2 b$ and $3 b$ ). More extensive global sampling is required to confirm the generality of this latitudinal geographic structure to genetic diversity in C. briggsae.

\section{Vulval cell lineage}

In $C$. elegans, the significant variation we observed among strains in the division frequency of the P3.p vulval precursor cell $\left(\chi_{19}^{2}=447.5, P<0.0001\right)$ indicates that considerable heritable phenotypic diversity exists in the African sample, despite a lack of substantial genetic variation at the loci surveyed here (Figure 4). For example, ED3046 (South Africa) and ED3054 (Kenya) exhibited a 38\% difference in P3.p division frequency, yet differed by only one SNP across the $\sim 4-\mathrm{kb}$ molecular dataset (see Figure 2a). Unlike the variation known for P3.p cell fate, the P4.p cell was previously thought to be largely invariant in the Caenorhabditis genus (Delattre and Félix, 2001); however, we found significant variation in 

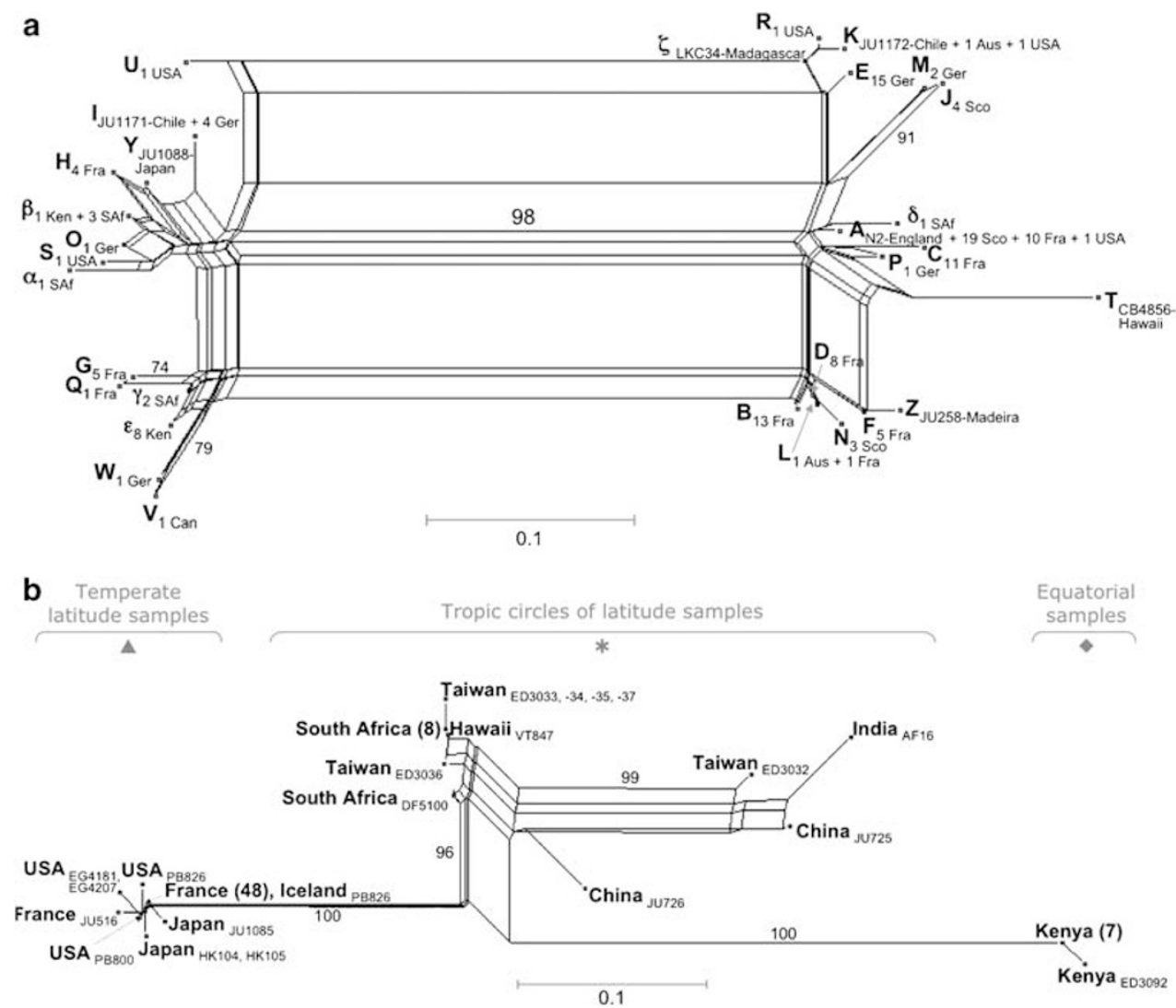

Tropic circles of latitude samples

*

Țaiwan ED3033,-34.-35.-37

Figure 3 Unrooted p-distance neighbor-nets for C. elegans (a) and C. briggsae (b) based on concatenated multilocus haplotypes. C. elegans haplotype designations are as shown in Figure 2a; African haplotypes are designated by Greek letters; subscripts indicate the number of strains per haplotype and the country of origin (Aus, Australia; Can, Canada; Fra, France; Ger, Germany; Ken, Kenya; SAf, South Africa; Sco, Scotland). C. briggsae clades are labeled following the latitudinal clades with the symbols in parentheses corresponding to those used to represent the strains in Figure 1. Bootstrap values $\geqslant 70 \%$ of 1000 replicates are shown next to the branches. Neighbor-joining trees are shown in Supplementary Figure S1.

C. briggsae strains for both the P3.p $\left(\chi_{9}^{2}=33.0, P=0.0001\right)$ and P4.p cells $\left(\chi_{9}^{2}=94.2, P<0.0001\right)$. In the two South African C. briggsae strains tested (ED3082 and ED3087), P4.p failed to divide $23-24 \%$ of the time. This level was significantly greater than for any other $C$. briggsae strain (all pairwise tests, $P<10^{-8}$ ). In our screens of three other species in the Elegans group of the genus, we observed this level of P4.p nondivision only in C. briggsae's closest relative, C. sp. 5 (JU727, 67\% P4.p nondivision frequency, $n=69 ;$ SB378, 5\% P4.p nondivision frequency, $n=113$ ). The unusual P4.p cell-lineage features found in the South African C. briggsae strains highlights the potential for significant phenotypic differences still to be found in these isolates, despite a high sequence similarity with the northern tropical circles of latitude strains.

\section{Hybrid crosses}

Despite large differences in the molecular sequences of $C$. briggsae isolates from different parts of the world, we did not find any gross hybrid incompatibilities between African strains and strains from elsewhere, although there is substantial quantitative variation between strains for brood size and embryonic lethality (Figure 5). Comparisons of pure strain versus hybrid F1s, however, show some evidence of mid-parent heterosis (MPH) for brood size, in which the hybrids show increased levels over the mean of the two parents. MPH was significantly positive in three of eight crosses, and the mean MPH value across all the crosses was $16.7 \%$. The F1 hybrids of one cross, ED3083 (South Africa) $\times$ ED3034 (Taiwan), even had significantly greater brood sizes than either pure-strain parent, displaying best-parent heterosis $\left(t_{30}=2.07, P=0.047\right)$, although this appears to be due to a large reduction in the embryonic lethality in the hybrids, which is quite high for both pure strains (Figure 5).

We repeated the assays of brood size and embryonic lethality in pure-strain worms, and confirmed that these same strains displayed high proportions of dead embryos and reduced fecundities (results not shown). The low brood sizes in these strains was also independently observed elsewhere (D Denver, personal communication). In these strains (and others from Johannesburg, South Africa), we also observed that the worms suffered from pale and vacuolated intestines (Supplementary Figure S2). This phenotype could result from an intracellular pathogen of intestinal cells, yet it was resistant to bleaching with alkaline hypochlorite-a method that is known to kill many extracellular pathogens, and eliminated an intracellular bacterium previously described in C. elegans (Barrière and Félix, 2005). 

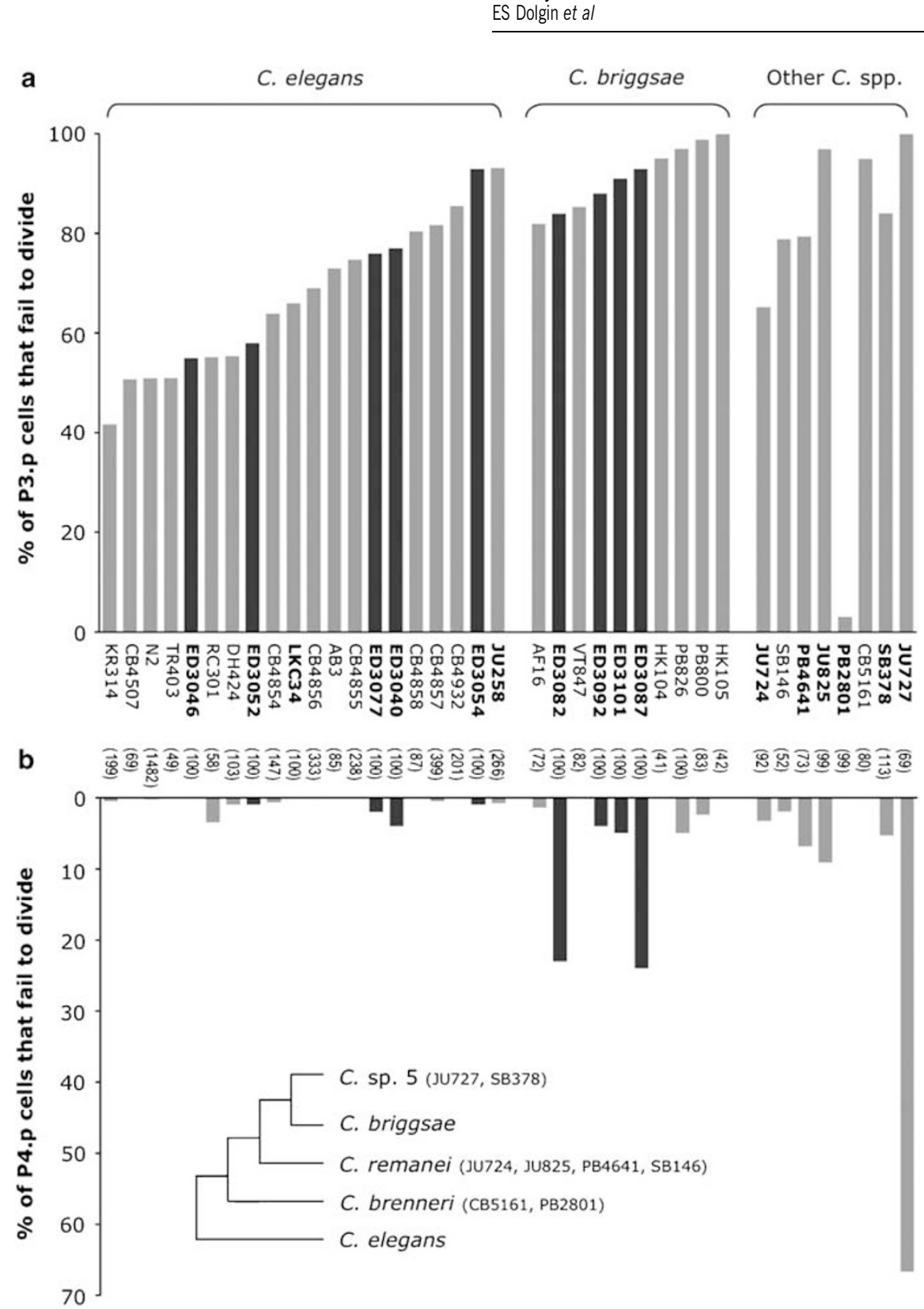

Figure 4 Percentage of P3.p (a) and P4.p (b) nondivision in hermaphrodites of C. elegans, C. briggsae and three other Caenorhabditis species of the Elegans group of the genus. The strain names in bold denote strains not previously analyzed by Delattre and Félix (2001). The darker shaded bars highlight the results for the newly collected African isolates. The number of observed worms is shown next to the strain name. The phylogenetic topology of Caenorhabditis species follows Kiontke and Fitch (2005) with C. sp. 5 added according to K Kiontke and D Fitch (personal communication).

This raises the possibility of a genetic basis to these traits, and more work is needed to explain the abnormal intestines and poor performance of these worms.

We also monitored the duration of development from egg to L4-adult molt in the pure strain and hybrid F2s, but only observed large numbers of delayed F2s in one class of worms-the hybrids of AF16 (India) and HK104 (Japan). There was a significant difference between the duration of development of AF16 $\times$ HK104 hybrids and pure strains $\left(\chi_{1}^{2}=33.7, P<0.0001\right)$, with $\sim 20 \%$ of hybrid F2s exhibiting delayed development. This confirms previous observations (S Baird, unpublished data), and suggests that the effect is peculiar to these genotypes, since this phenomenon was not found in any other crosses involving either the African isolates (results not shown) or other crosses without AF16 (S Baird, personal communication).

\section{Discussion}

The discovery of C. elegans and C. briggsae in sub-Saharan Africa, as well as additional strains from around the world, reinforces the notion that these are indeed cosmopolitan species and offers some novel insights about levels and patterns of global variation. In $C$. elegans, this study confirms the low levels of overall silent-site polymorphism previously found in Europe for another part of the world (Cutter, 2006). In C. briggsae, 

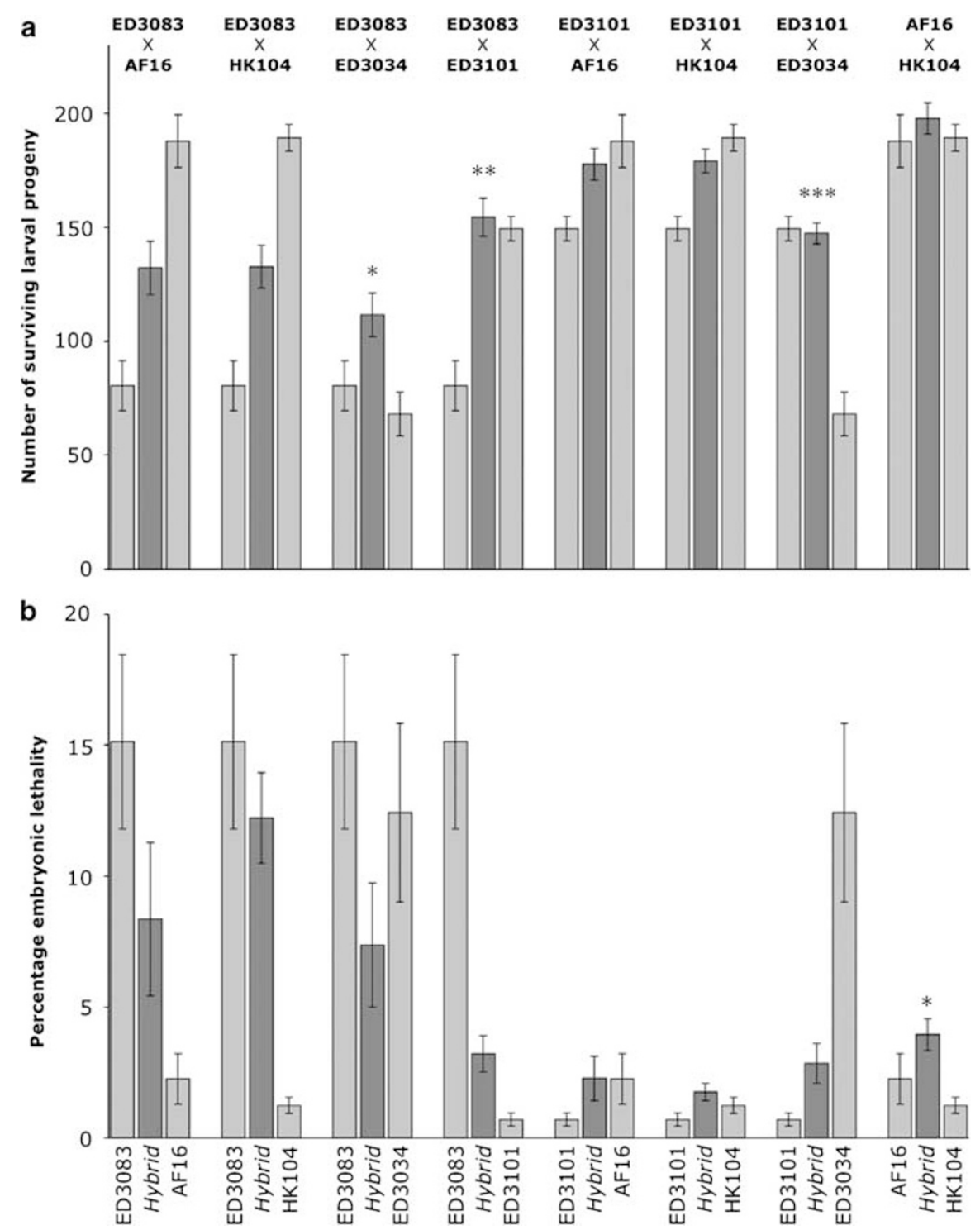

Figure 5 Surviving larval brood sizes (a) and embryonic lethalities (b) in the C. briggsae hybrid crosses. Pure strains (lightly shaded bars) are shown next to the hybrids (darkly shaded bars) in each cross. For ease of comparison, identical data for pure strains is shown multiple times. Asterisks $\left.{ }^{*}\right)$ indicate significance in a $t$-test of the deviation between hybrid and mid-parent. ${ }^{*} P<0.05,{ }^{* *} P<0.01,{ }^{* * *} P<0.001$. Error bars indicate \pm 1 s.e.

Kenyan samples raise global species diversity estimates as a consequence of between population differences, despite a lack of variation within each of the two sampling sites in Africa. This result is similar to the only other known study that has established multiple wild strains from single locations (Cutter et al., 2006b), although whether this reflects true levels of withinpopulation variation in C. briggsae or a biased local sampling procedure remains to be seen. The natural habitat of C. elegans and C. briggsae is unknown, although both species are routinely found in anthropogenic habitats, such as compost and garden soil, and they often form phoretic and/or necromenic associations with other invertebrates (Kiontke and Sudhaus, 2006). Given their apparent similarity in sampled habitats, it is striking that the two species have such different geographic patterning of molecular diversity (see
Figure 3), even more so considering that $C$. elegans and C. briggsae were found co-occurring in the same sampling sites in Kenya and South Africa, as well as France (Barrière and Félix, 2005) and Japan (JU1085 and JU1088, described in this study). This begs the question of what could have led to the different biogeographic structures in the two species.

Population genetic studies in C. elegans have demonstrated that migration occurs over surprisingly large distances, including continents (Haber et al., 2005; Cutter, 2006; Barrière and Félix, 2005, 2007). Since the latitudinal clades we observe appear circumnavigatory, this may be true of $C$. briggsae as well. It has been proposed that dispersion in these species might be facilitated by human activities (Kiontke and Sudhaus, 2006), but it is conceivable that the migration patterns and the role of human-mediated dispersion in the two species are quite 
different. Although both species co-occur in some localities, there may be substantial differences in their natural ecology that we have failed to appreciate. Another possibility is that $C$. briggsae more recently colonized different parts of the world than $C$. elegans (Cutter et al., 2006b). However, our evidence of a third distinct geographic haplotype group in equatorial Kenya with many unique polymorphisms makes it difficult to determine the source of such a founder event. Furthermore, the lack of additional recombination events detected from the inclusion of Kenyan isolates implies that these populations might be isolated relative to other known samples. In C. elegans, two strains in particular (CB4856/Hawaii and JU258/Madeira), both from remote island locations, have been shown to be highly divergent from most other strains (Koch et al., 2000; Haber et al., 2005; Stewart et al., 2005; although across the six loci tested here, we did not observe this for JU258, see Figures $2 \mathrm{a}$ and $3 \mathrm{a}$ ), but it seems unlikely that samples from urbanized areas of Africa will be as isolated as these island strains. The absence of detectable recombination between the three clades in C. briggsae suggests that migration patterns and outcrossing rates could indeed be quite different in C. briggsae and C. elegans. This is further indicated by the lack of within-locality diversity in Kenya, South Africa and France (Cutter et al., 2006b), which implies that gene flow is not sufficiently prevalent to prevent structuring by genetic drift and/or local selection, and is qualitatively consistent with a lower rate of outcrossing in C. briggsae (Cutter et al., 2006b).

Our evidence for three distinct latitudinal haplotype groups in C. briggsae, including a 'Tropic circles of latitude' clade that spans both hemispheres, suggests that ecological factors might also play a role in driving the observed patterns of biogeographic structure. One might speculate that this geographical partitioning of haplotypes reflects local adaptation. One possible outcome of this scenario would be outbreeding depression. However, we observed marginal inbreeding depression in the hybrid crosses between different latitudinal clades. In fact, outbreeding depression was previously observed for $C$. elegans, although this was probably due to the effects of the hybrid breakdown of coadapted gene complexes, rather than to effects of local adaptation (Dolgin et al., 2007). Thus, it may be the case that local inbreeding and genetic drift are sufficient to explain the inbreeding depression observed between disparate population samples of $C$. briggsae. A possible methodological explanation for the inbreeding depression is that we performed our hybrid crosses under standard laboratory conditions of $20^{\circ} \mathrm{C}$, and inbreeding depression was primarily found for crosses involving ED3101 from Nairobi, Kenya. Considering that the average monthly maximum ambient temperature in Nairobi is never this low, these conditions might constitute a stressful environment for this strain, resulting in inbreeding depression (Armbruster and Reed, 2005), whereas experimentation under more benign conditions could produce different results.

Even though C. elegans displays low levels of molecular variation, wild isolates show phenotypic variation in many potentially ecologically relevant traits, including dauer sensitivity (Viney et al., 2003), response to pathogens (Schulenburg and Müller, 2004), clumping behavior (Hodgkin and Doniach, 1997; de Bono and
Bargmann, 1998) and locomotory speed (de Bono and Bargmann, 1998). Relating these phenotypes to their ecological importance, however, remains elusive. The low degree of variation and extensive linkage disequilibrium found in $C$. elegans make it difficult to detect signatures of local adaptation using traditional molecular population genetics approaches. The same is true for C. briggsae, but the more structured differences in genetic variation among geographic regions in C. briggsae might allow laboratory approaches, such as quantitative trait loci (QTL) mapping, to identify regions of the genome that are associated with phenotypic differences between strains from different parts of the world. In C. elegans, QTLs have been identified for a number of life-history related traits, with evidence of genotype-environment interactions, including in response to temperature (Shook and Johnson, 1999; Knight et al., 2001; Ayyadevara et al., 2003; Gutteling et al., 2007). However, the lack of correlation between biogeographic structure and diversity in $C$. elegans means it is difficult to make inferences about environmental adaptations, and suggests that any QTL identified might not necessarily correlate neatly with ecology.

The biogeographic patterning of C. briggsae could make this species more amenable than $C$. elegans to studies of ecological and behavioral adaptation (Baird and Chamberlin, 2006; Cutter et al., 2006b). The few studies that examined strain-specific differences in C. briggsae have shown that strains from different clades exhibit variation in hybrid compatibilities (Baird, 2002), the patterning of sensory rays in the male tail (Baird, 2001) and vulval cell lineages (Delattre and Félix, 2001). In this study, we demonstrated that additional phenotypic variation is also found within clades, as South African C. briggsae strains exhibited a unique pattern of P4.p division despite quite similar molecular sequences to strains from the Tropic of Cancer. It remains to be seen, however, how distinct the genomes of this particular set of strains are, and how much within- and between-clade diversity exists in the species. More samples will also be needed to determine if the latitudinal clade pattern found here is a common global feature, and to more accurately determine how the patterns of migration, outcrossing and selection differ between $C$. elegans and C. briggsae. With a growing number of strains being collected from around the world, and increasing genetic and molecular resources, $C$. briggsae presents itself as a useful companion species to C. elegans, and might be well suited to studying some questions of gene flow, speciation and adaptation.

\section{Acknowledgements}

We are grateful to $\mathrm{M}$ Owen-Smith, A Müller and $\mathrm{K}$ Amalemba for assistance with field collections, and to C Griffiths and I Gordon for providing laboratory facilities. Worm strains were generously provided by L Carta, K Kiontke and the Caenorhabditis Genetics Center. We thank B Charlesworth and three anonymous reviewers for helpful comments on the manuscript, and to $\mathrm{M}$ Ailion, $\mathrm{S}$ Baird and $\mathrm{D}$ Denver for discussing unpublished results. This work was funded by a University of Edinburgh Development Trust Small Project Grant, and by postgraduate scholarships from the Natural Sciences and Engineering Research Council 
(Canada) and the University of Edinburgh School of Biological Sciences to ESD, and by the National Science Foundation (USA) with International Research Fellowship Program grant 0401897 to ADC.

\section{References}

Armbruster P, Reed DH (2005). Inbreeding depression in benign and stressful environments. Heredity 95: 235-242.

Ayyadevara S, Ayyadevara R, Vertino A, Galecki A, Thaden JJ, Shmookler Reis RJ (2003). Genetic loci modulating fitness and life span in Caenorhabditis elegans: categorial trait interval mapping in CL2a $x$ Beregerac-BO recombinant-inbred worms. Genetics 163: 557-570.

Baïlle D, Barrière A, Félix M-A (2008). Oscheius tipulae, a widespread hermaphroditic soil nematode, displays a higher genetic diversity and geographic structure than Caenorhabditis elegans. Mol Ecol, in press.

Baird SE (2001). Strain-specific variation in the pattern of caudal papillae in Caenorhabditis briggsae (Nematoda: Rhabditidae); implications for species identification. Nematology 3 : 373-376.

Baird SE (2002). Haldane's rule by sexual transformation in Caenorhabditis. Genetics 161: 1349-1353.

Baird SE, Chamberlin HM (2006). Caenorhabditis briggsae methods (December 18, 2006). In: The C. elegans Research Community (ed). Wormbook, doi/10.1895/wormbook.1.128.1, http:/ / www.wormbook.org.

Baird SE, Davidson CR, Bohrer JC (2005). The genetics of ray pattern variation in Caenorhabditis briggsae. BMC Evol Biol 5: 3 .

Barrière A, Félix M-A (2005). High local genetic diversity and low outcrossing rate in Caenorhabditis elegans natural populations. Curr Biol 15: 1176-1184.

Barrière A, Félix M-A (2006). Isolation of C. elegans and related nematodes (July 17, 2006). In: The C. elegans Research Community (ed). Wormbook, doi/10.1895/wormbook.1.115.1, http:/ / www.wormbook.org.

Barrière A, Félix M-A (2007). Temporal dynamics and linkage disequilibrium in natural Caenorhabditis elegans populations. Genetics 176: 999-1011.

Charlesworth D (2003). Effects of inbreeding on the genetic diversity of populations. Philos Trans $R$ Soc Lond B Biol Sci 358: 1051-1070.

Coghlan A, Wolfe KH (2002). Fourfold faster rate of genome rearrangement in nematodes than in Drosophila. Genome Res 12: $857-867$.

Cutter AD (2006). Nucleotide polymorphism and linkage disequilibrium in wild populations of the partial selfer Caenorhabditis elegans. Genetics 172: 171-184.

Cutter AD, Baird SE, Charlesworth D (2006a). High nucleotide polymorphism and rapid decay of linkage disequilibrium in wild populations of Caenorhabditis remanei. Genetics $\mathbf{1 7 4}$ 901-913.

Cutter AD, Félix M-A, Barrière A, Charlesworth D (2006b). Patterns of nucleotide polymorphism distinguish temperate and tropical wild isolates of Caenorhabditis briggsae. Genetics 173: 2021-2031.

de Bono M, Bargmann CI (1998). Natural variation in a neuropeptide $\mathrm{Y}$ receptor homolog modifies social behavior and food response in C. elegans. Cell 94: 679-689.

Delattre M, Félix M-A (2001). Polymorphism and evolution of vulval precursor cell lineages within two nematode genera, Caenorhabditis and Oscheius. Curr Biol 11: 631-643.

Denver DR, Morris K, Thomas WK (2003). Phylogenetics in Caenorhabditis elegans: an analysis of divergence and outcrossing. Mol Biol Evol 20: 393-400.

Dolgin ES, Charlesworth B, Baird SE, Cutter AD (2007). Inbreeding and outbreeding depression in Caenorhabditis nematodes. Evolution 61: 1339-1352.
Floyd R, Abebe E, Papert A, Blaxter M (2002). Molecular barcodes for soil nematode identification. Mol Ecol 11: 839-850.

Fodor A, Riddle DL, Nelson FK, Golden JW (1983). Comparison of a new wild-type Caenorhabditis briggsae with laboratory strains of Caenorhabditis briggsae and C. elegans. Nematologica 29: 203-217.

Graustein A, Gaspar JM, Walters JR, Palopoli MF (2002). Levels of DNA polymorphism vary with mating system in the nematode genus Caenorhabditis. Genetics 161: 99-107.

Gutteling EW, Riksen JAG, Bakker J, Kammenga JE (2007). Mapping phenotypic plasticity and genotype-environment interactions affecting life-history traits in Caenorhabditis elegans. Heredity 98: 28-37.

Haag ES, Ackerman AD (2005). Intraspecific variation in fem-3 and tra-2, two rapidly coevolving nematode sex-determining genes. Gene 349: 35-42.

Haber M, Schüngel M, Putz A, Müller S, Hasert B, Schulenburg H (2005). Evolutionary history of Caenorhabditis elegans inferred from microsatellites: evidence for spatial and temporal genetic differentiation and the occurrence of outbreeding. Mol Biol Evol 22: 160-173.

Hillier LW, Miller RD, Baird SE, Chinwalla A, Fulton LA, Koboldt DC et al. (2007). Comparison of C. elegans and C. briggsae genome sequences reveals extensive conservation of chromosome organization and synteny. PLoS Biol 5: e167.

Hodgkin J, Doniach T (1997). Natural variation and copulatory plug formation in Caenorhabditis elegans. Genetics 146: 149-164.

Hudson RR (2000). A new statistic for detecting differentiation. Genetics 155: 2011-2014.

Hudson RR, Boos DD, Kaplan NL (1992). A statistical test for detecting geographic subdivision. Mol Biol Evol 9: 138-151.

Hudson RR, Kaplan NL (1985). Statistical properties of the number of recombination events in the history of a sample of DNA sequences. Genetics 111: 147-164.

Huson DH, Bryant D (2006). Applications of phylogenetic networks in evolutionary studies. Mol Biol Evol 23: 254-267.

Inoue T, Ailion M, Poon S, Kim HK, Thomas JH, Sternberg PW (2007). Genetic analysis of dauer formation in Caenorhabditis briggsae. Genetics 177: 809-818.

Jovelin R, Ajie BC, Phillips PC (2003). Molecular evolution and quantitative variation for chemosensory behaviour in the nematode genus Caenorhabditis. Mol Ecol 12: 1325-1337.

Kiontke K, Fitch DH (2005). The phylogenetic relationships of Caenorhabditis and other rhabditids (August 11, 2005). In: The C. elegans Research Community (ed). Wormbook, doi/ 10.1895/wormbook.1.11.1, http:/ / www.wormbook.org.

Kiontke K, Gavin NP, Raynes Y, Roehrig C, Piano F, Fitch DH (2004). Caenorhabditis phylogeny predicts convergence of hermaphroditism and extensive intron loss. Proc Natl Acad Sci USA 101: 9003-9008.

Kiontke K, Sudhaus W (2006). Ecology of Caenorhabditis species (January 9, 2006). In: The C. elegans Research Community (ed). Wormbook, doi/10.1895/wormbook.1.37.1, http:// www.wormbook.org.

Knight CG, Azevedo RBR, Leroi AM (2001). Testing life-history pleiotropy in Caenorhabditis elegans. Evolution 55: 1795-1804.

Koch R, van Luenen HGAM, van der Horst M, Thijssen KL, Plasterk RHA (2000). Single nucleotide polymorphisms in wild isolates of Caenorhabditis elegans. Genome Res 10: 1690-1696.

Maupas EF (1900). Modes et formes de reproduction des nématodes. Arch Zool Expér Gén 8: 463-624.

Maydan JS, Flibotte S, Edgley ML, Lau J, Selzer RR, Richmond TA et al. (2007). Efficient high-resolution deletion discovery in Caenorhabditis elegans by array comparative genomic hybridization. Genome Res 17: 337-347. 
Nigon V, Dougherty EC (1949). Reproductive patterns and attempts at reciprocal crossing of Rhabditis elegans, and Rhabditis briggsae Dougherty and Nigon, 1949 (Nematoda: Rhabditidae). J Exp Zool 112: 485-503.

Rozas J, Sanchez-DelBarrio JC, Messeguer X, Rozas R (2003). DnaSP, DNA polymorphism analyses by the coalescent and other methods. Bioinformatics 19: 2496-2497.

Schulenburg H, Müller S (2004). Natural variation in the response of Caenorhabditis elegans towards Bacillus thuringiensis. Parasitology 128: 433-443.

Shook DR, Johnson TE (1999). Quantitative trait loci affecting survival and fertility-related traits in Caenorhabditis elegans show genotype-environment interactions, pleiotropy and epistasis. Genetics 153: 1233-1243.
Sokal RR, Rohlf FJ (1995). Biometry. W.H. Freeman and Company: New York.

Stein LD, Bao Z, Blasiar D, Blumenthal T, Brent MR, Chen $N$ et al. (2003). The genome sequence of Caenorhabditis briggsae: a platform for comparative genomics. PLoS Biol 1: e45.

Stewart MK, Clark NL, Merrihew G, Galloway EM, Thomas JH (2005). High genetic diversity in the chemoreceptor superfamily of Caenorhabditis elegans. Genetics 169: 1985-1996.

Tajima F (1989). Statistical method for testing the neutral mutation hypothesis. Genetics 123: 585-595.

Viney ME, Gardner MP, Jackson JA (2003). Variation in Caenorhabditis elegans dauer larva formation. Dev Growth Differ 45: 389-396.

Supplementary Information accompanies the paper on Heredity website (http://www.nature.com/hdy) 